\title{
¿DEBEN LOS FILÓSOFOS MORALES APRENDER DE LOS JURISTAS?*
}

\author{
Hugo Seleme \\ Universidad Nacional de Córdoba \\ CONICET
}

RESUMEN. Existen diferentes servicios que los filósofos morales pueden prestarles a los juristas. Los mismos son variados y ha sido explicitados en diferentes trabajos. La hipótesis de este trabajo es que la relación inversa también es cierta. Existen tres maneras viciadas de hacer filosofía moral que los juristas pueden ayudar a combatir. La primera consiste en la obsesión metaética fundada en un sesgo escéptico hacia cualquier reflexión sustantiva. La segunda es el diletantismo activista de aquellos que sin ninguna preparación teórica se dedican a hablar de problemas morales y políticos. La tercera consiste en una forma de reflexión filosófica que en su afán de encontrar los principios supremos ha perdido todo contacto con la experiencia moral humana. El contacto con los juristas puede ayudar a combatir estos vicios, prestando de este modo la reflexión jurídica un servicio a la Filosofía Moral.

Palabras clave: Filosofía Moral Viciada, Filosofía del derecho, Metaética, Experiencia moral humana.

\section{Should moral philosophers learn from lawyers?}

ABSTRACT. Moral philosophy may help Jurisprudence in several forms. The services that Moral Philosophy may offer to Jurisprudence have been explored in different works. This paper advances the hypothesis that the inverse relation is also true. There are three flawed ways of doing moral philosophy that lawyers can help fight. The first is the meta ethics obsession based on a skeptical bias against any substantive reflection. The second is the activist dilettantism of those who talk about moral and political issues without having any theoretical background. The third is a form of philosophical reflection that on the way to looking for the supreme principles of morality it has lost touch with any human moral experience. Contact with lawyers can help fight these vices. This is a service that legal reflection may offer to Moral Philosophy.

Keywords: Flawed Moral Philosophy, Jurisprudence, Metaethics, Moral human experience.

* Fecha de recepción: 31 de marzo de 2014. Fecha de aceptación: 22 de mayo de 2014.

Agradezco los comentarios y sugerencias de Sebastián AlOnSo AgÜERO, Alberto CARRIO, Marisa IgLESIAS, Jorge MALEM, José Luis MARTí, José Juan MORESO y Jahel Queralt. 


\section{LA FILOSOFÍA MORAL Y EL DERECHO}

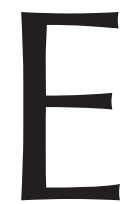

111 de octubre del año 2000 el New York Council for the Humanities le confirió a Ronald Dworkin la distinción de Scholar of the Year. En es evento Dworkin ofreció una conferencia titulada «Must Our Judges Be Philosophers? Can They Be Philosophers?» (DwORKIN, 2000). La posición que presentó en esa oportunidad es una que ha ido ganando consenso en nuestro entorno académico ${ }^{1}$. La misma sostiene que los juristas abordan problemas que han sido tratados de manera detallada por los filósofos y que, por tanto, harían bien en prestar atención a lo que éstos han dicho.

Los conceptos que son utilizados por los juristas - y los asuntos a los que hacen referencia - son semejantes o idénticos a los que han ocupado a los filósofos morales. Tanto unos como otros están preocupados por identificar en qué consiste la igualdad, la libertad, la responsabilidad, la democracia o la intencionalidad. Los juristas tienen que embarcarse en una tarea que es parcialmente semejante a la que han llevado adelante por siglos los filósofos morales y es lógico y deseable esperar que se beneficien del conocimiento y la experiencia que éstos han acumulado.

Aunque la recomendación de DwORKIN a los juristas se asienta en su particular concepción del derecho, según la cual es necesario ofrecer una lectura moral de la Constitución, no es una tesis que sólo pueda ser defendida sobre estas bases y por la que sólo él haya abogado. Marta NusSBAUM, por caso, ha sostenido una posición semejante sin tener una concepción acerca del derecho que sea semejante a la de DwORKIN (Nussbaum, 1993).

Según Nussbaum los usos que la filosofía puede tener para los juristas son variados. La Filosofía no sólo ha trabajado sobre los mismos conceptos que utilizan los juristas - entre los que ella destaca los conceptos de libre albedrío, emoción, sexualidad y calidad de vida - sino que también puede aportar claridad en cuestiones metodológicas y epistemológicas, ofrecer soluciones a problemas políticos concretos tales como los vinculados, por ejemplo, con la regulación de la profesión médica, investigar sobre la naturaleza de los sistemas jurídicos — que es lo que mayormente han hecho los filósofos del derecho- y ayudar a que los juristas mantengan vivo su afán por seguirse cuestionando acerca de las cuestiones controvertidas en lugar de intentar darles una rápida solución.

Hasta alguien tan lejano a la concepción del derecho defendida por DwORKIN, como Richard POSNER, parece acordar en que las herramientas filosóficas son de utilidad para el jurista. Según POSNER «...las técnicas de la filosofía analítica y las del razonamiento jurídico son similares...» (POSNER, 1993: 465). Sin embargo, POSNER piensa que los juristas se han mostrado más capaces de apropiarse de los desarrollos de la filosofía, que lo que han sido los filósofos de interiorizarse en los asuntos jurídicos. Por esta razón considera, a diferencia de NusSBAUM, que no existe ningún aporte relevante que los filósofos puedan realizar en las escuelas de derecho. Lo que diferencia a Posner de Nussbaum y Dworkin no es la idea de que la Filosofía tenga aportes que

${ }^{1}$ Me refiero a las escuelas de derecho con una fuerte tradición ius-positivista de España, Italia y Latinoamérica. 
realizar al derecho, sobre lo que acuerdan, sino el que los filósofos tengan algo que aportar $^{2}$.

La idea que los juristas deben aprender de la Filosofía tampoco está circunscrita en el tiempo. No se trata de una idea que haya sido introducida recientemente por autores contemporáneos como los que he señalado. NussBAUM se ha encargado de señalar cómo la misma idea podía encontrarse ya, aunque con diferentes matices, en SÓCRATES y ARISTÓTELES. Para el primero, el filósofo debía tomar las concepciones elaboradas por los juristas y los funcionarios en general, con el objeto de dotarlas de mayor rigor y claridad. Este en última instancia era el fin que perseguía el método mayeútico. ARISTÓTELES, por su parte, compartía esta visión de la filosofía como herramienta para alcanzar la claridad y el rigor conceptual (NusSBAUM, 1993: 1628).

Esta convicción de que la Filosofía —al menos la corriente analítica- tiene algo para aportar a los juristas tampoco es algo que haya sido señalado sólo por filósofos o juristas del pasado o del presente. Recientemente ha sido refrendada por la American Bar Association. Dicha Asociación a comienzo del año 1989 estableció, a través del Consejo de la Sección de Educación Jurídica y Admisión al Ejercicio Profesional, la denominada «Fuerza Operativa». Su objetivo era desarrollar un Listado de Habilidades y Valores que un abogado debería tener y, por tanto, una escuela de derecho debería transmitir. En 1992 - luego de más de dos años de estudio— la «Fuerza Operativa» publicó un listado de diez habilidades que todo abogado debía poseer. La lista incluía habilidades que se encuentran vinculadas con el pensamiento filosófico en general - como la referida a la resolución de problemas o el análisis - pero también habilidades vinculadas de modo expreso con la Filosofía Moral. En este sentido, se recomendaba que los abogados desarrollasen las «...habilidades involucradas en el reconocimiento y resolución de dilemas éticos» (ABA, Section of Legal Education and Admission to the Bar, 1992: $135)^{3}$. Al explicar esta habilidad agrega que los abogados debían conocer «...(a)spects of ethical philosophy bearing upon the propriety of particular practices or conduct (such as, for example, general ethical precepts calling for honesty, integrity, courtesy and respect for others; general ethical probibitions against lying and misrepresentation)...» (ABA, Section of Legal Education and Admission to the Bar, 1992: 204).

La convicción de que la Filosofía en general, y la Filosofía Moral en particular, tiene algo que aportar al derecho se ha extendido durante los últimos años no sólo entre los juristas sino también entre los abogados. Esto ha tenido el efecto benéfico de favorecer una mayor interdisciplinariedad en la enseñanza del derecho. Las escuelas de derecho han abierto sus puertas a prestigiosos filósofos morales, y juristas y abo-

2 POSNER piensa que los estudiantes que demuestren talento para la filosofía se verán naturalmente atraídos a seguir la carrera de derecho dado que se trata del mismo talento requerido para ser abogado y la profesión de abogado está mucho mejor pagada que la de filósofo. Señala al respecto: «... A college student who bas done well in philosophy and thinks he might like to apply philosophy to law has a reasonable expectation of doing well in law school, and a law degree is the only graduate degree he needs in order to become a law professor at twice the salary a philosophy professor can command or to become a legal practitioner at several times the salary that either can command...» (POSNER, 1993: 465).

3 El reporte que contenía el Listado de Habilidades y Valores sostenía que «In order to represent a client consistently with applicable ethical standards, a lawyer should be familiar with: the nature and sources of ethical standards; the means by which ethical standards are enforced; the processes for recognizing and resolving ethical dilemmas» (ABA, Section of Legal Education and Admission to the Bar, 1992: 140). 
gados se han dispuesto a aprender el manejo de las herramientas éticas en las que los filósofos aparecían como especialistas. Juristas y abogados se han ubicado en el lugar de alumnos esperando escuchar lo que los filósofos morales tenían para decir sobre los problemas acuciantes que el derecho enfrenta, tales como el aborto, la eutanasia o las manos sucias. La experiencia no ha sido siempre feliz.

En parte esto se ha debido a la urgencia de los juristas y abogados por encontrar soluciones a los problemas prácticos que enfrentan. Éstos perciben que tienen una responsabilidad frente a sus clientes y a la sociedad en general. Tienen que decidir — si son jueces - argüir — si son litigantes — o aconsejar — si son asesores- sobre qué se debe hacer. Esto lleva a que tengan una tendencia a preocuparse más por encontrar la solución a los problemas prácticos que a intentar entenderlos en toda su complejidad. Los abogados y juristas, para decirlo de modo gráfico, se impacientan frente a filósofos morales que encuentran mayor deleite en presentar problemas que en encontrar soluciones. Piensan que los filósofos morales pueden darse el lujo de la reflexión sin fin sólo porque no poseen ninguna responsabilidad práctica. Los filósofos morales pueden darse el lujo de cavilar indefinidamente porque no tienen urgencia de decidir.

Judith Jarvis ThOMson, una de las filósofas morales contemporáneas más prestigiosas tuvo ocasión de padecer en primera persona la impaciencia de los abogados y juristas. Invitada a dictar un curso en la escuela de derecho de Yale, decidió explicar a sus alumnos sus famosos «trolley cases». La idea de estos experimentos mentales es mostrar lo dificultoso que es acomodar nuestras intuiciones morales sobre matar por acción u omisión, consecuencias queridas o meramente previstas, responsabilidad directa o indirecta, etc. La complejidad se advierte al mostrar que los casos llevan aparentemente a dilemas sin salida. THOMSON no tuvo éxito en mostrar la complejidad del problema a sus alumnos porque éstos en lugar de detenerse a advertir los problemas que los casos presentaban, una y otra vez le exigían a ella que les dijese cuál era la respuesta correcta ${ }^{4}$.

Las anécdotas podrían multiplicarse y cualquiera que haya estado en contacto con filósofos morales que hayan pasado por una escuela de derecho, habrá escuchado alguna versión de la misma. Los abogados y los juristas están más preocupados por tomar nota de las soluciones que en reflexionar sobre los problemas. Aunque pienso que esto tiene algo de cierto, creo que no es la historia completa. Creo que existe algo que los filósofos morales deben aprender de los juristas y que harían mal en pasar por alto su impaciencia desdeñándola como mero producto del carácter irreflexivo de los abogados. Creo también que los abogados y juristas harían bien en no suscribir el diagnóstico autocomplaciente ofrecido por los filósofos morales, según el cual el problema radica en la falta de capacidad para ascender a las alturas de la reflexión filosófica.

En lo que sigue pretendo contribuir a que esto suceda. En primer lugar, intentaré mostrar que puede existir otra explicación de la impaciencia de los juristas. Sugeriré que ésta tiene más que ver con los defectos de cierto modo de hacer Filosofía Moral

4 ThOmSON contó esta experiencia a NussBaum, y es esta última quien hace referencia a la misma (Nussbaum, 1993: 1640). 
que con la falta de carácter reflexivo de los juristas. Espero que esto contribuya a paliar el sentimiento de culpa y minusvalía con el que los juristas abandonan los recintos donde los filósofos morales han intentado iluminarlos, infructuosamente, con su saber.

En segundo lugar, señalaré que la posición de responsabilidad en la que se encuentran los juristas - apremiados por brindar soluciones a problemas concretos- que puede conducirlos a no detenerse a reflexionar, también puede facilitarles una mejor comprensión de los dramas humanos que subyacen a los problemas morales. El tipo de reflexión responsable, enraizada en la experiencia, en la que los juristas son educados puede ayudar a corregir la futilidad de cierta Filosofía Moral orgullosamente desencajada de la experiencia y soberanamente independiente de cualquier responsabilidad práctica. Espero que esto contribuya a que los filósofos morales perciban la contribución que la reflexión jurídica puede brindarles, volviendo a conectar sus elucubraciones teóricas con la experiencia humana.

Después de todo, tal vez exista algo que los filósofos morales deban aprender de los juristas. Tal vez existe un lugar para los juristas en las escuelas de filosofía y no sólo un lugar para los filósofos en las escuelas de derecho. Quizás no sólo los juristas deban aprender de los filósofos morales sino, como el mismo DwORKIN pensaba, también los filósofos morales deban sentarse en el lugar de alumnos y escuchar lo que los juristas tienen para decir.

\section{UNA FILOSOFÍA MORAL VICIADA}

La anécdota de Thomson muestra la mala cara de la impaciencia de los juristas. THOMSON es una filósofa moral sutil, profundamente sensible a la experiencia moral y los juristas hubiesen hecho bien en tener la paciencia suficiente para apreciar la magnitud de los problemas que tenía para señalarles. Sin embargo, no todos los casos son como el de THOMSON. En una oportunidad asistí a un seminario donde el ponente concluyó, luego de veinte minutos de exposición, que existía un deber moral de abortar mientras hubiese un ser sintiente cuyo sufrimiento pudiésemos aliviar con los recursos que insumimos en el feto. En otra, la conclusión de la ponencia era que existía un deber moral de hacer que se extinguiesen los animales carnívoros para evitar el sufrimiento de los animales que les sirven de alimento. Y la lista podría continuar.

El estupor y la impaciencia de los juristas frente a estas afirmaciones deberían ser distinguidas cuidadosamente de la impaciencia de los estudiantes de THOMSON. En ambos casos la impaciencia se explica por un vicio, pero mientras en el caso de THOMSON el vicio residía en el carácter irreflexivo de sus alumnos, en el segundo tipo de casos lo viciado es el modo de hacer Filosofía Moral que ejemplifican. No hay nada que los juristas deban corregir o revisar en su reacción negativa frente a este modo viciado de hacer Filosofía Moral.

Creo que lo peor que podría suceder es que los juristas no distingan entre ambos modos de hacer Filosofía Moral. La falta de discernimiento puede conducir a dos resultados indeseables: que los juristas piensen que, dado que su actitud frente a posiciones como la de THOMSON era injustificada, lo mismo debe suceder con su impa- 
ciencia y desazón frente a quienes afirman que, por ejemplo, existe el deber general de abortar o el de extinguir especies de animales; o que piensen que, dado lo justificado que está su impaciencia frente a ese segundo tipo de Filosofía Moral, entonces no hay nada sensato que puedan aprender de esta disciplina. Es decir, que concluyan que su impaciencia frente a filósofos como THOMSON está justificada.

Es importante, entonces, identificar cuáles son los vicios que pueden aquejar a la Filosofía Moral. En lo que sigue, basándome en lo señalado por Bernard WiLLiams, identificaré tres: la obsesión por la metaética, la militancia diletante y la insensibilidad a la experiencia moral. De los tres, por lejos, el más pernicioso es el último. Que haya sido un filósofo moral quien identificase los vicios de cierto modo de hacer Filosofía Moral es una muestra de que los juristas se apresurarían si condenasen a todos por los vicios de algunos o, a la inversa, pasasen por alto los vicios de algunos por las virtudes de otros.

En el prefacio de su libro Morality, Williams identifica el primer vicio de la Filosofía Moral en ese momento, esto es 1972. El problema consistía en que la Filosofía Moral no se ocupaba de problemas prácticos. Estaba dedicada a identificar el significado de los términos morales y se había transformado en algo así como una filosofía del lenguaje moral. De este modo de hacer Filosofía Moral focalizada en los problemas metaéticos WiLLIAMs decía: «Contemporary moral philosophy bas found an original way of being boring, which is by not discussing moral issues at all...» (WILLIAMS, 1972: xvii).

Este modo de hacer Filosofía Moral ha ganado espacio en las escuelas de derecho, a la par que lo iba perdiendo dentro de la propia Filosofía Moral. Tal vez haya contribuido a ello el escepticismo moral que caracterizó a cierto tipo de positivismo jurídico. Dado que el interés por el discurso moral no podía ser sustantivo — debido al sesgo escéptico- el único enfoque interesante que podía dársele era uno referido al significado de los términos morales. Otra razón puede encontrarse en el afán de los juristas por encontrar el significado de los términos utilizados en los textos legales. La preocupación por el significado ocupa un lugar central en el derecho, lo que puede explicar que su interés por la moral haya estado también centrado en el significado ${ }^{5}$.

\footnotetext{
5 Philip PETTIT ha identificado razones metodológicas y sustantivas para que los filósofos analíticos creyesen —incluido el positivismo jurídico analítico — que no existía nada de interés en la discusión de las cuestiones morales y políticas sustantivas y cayesen en lo que él denomina «the long silence».

Por lo que respecta a las razones metodológicas, PETTIT señala que durante los años veinte y treinta dos proposiciones pasaron a formar parte de la ortodoxia de la filosofía analítica: «One of these propositions was that evaluative or normative assertions did not serve, or at least did not serve primarily, to essay a belief as to how things are; their main job was to express emotion or approval/disapproval, much in the manner of an exclamation like "Wow!" or "Ugh!" The other proposition was that among assertions that do express belief, there is a fairly exact divide between empirical claims that are vulnerable to evidential checks and analytical or a priori claims, such as mathematical propositions, that are true in virtue of the meaning of their terms». Dado que la Filosofía Política —y la Filosofía Moral en general— no eran ni una disciplina empírica ni se ocupaban de encontrar verdades a priori, continúa PETTIT, entonces «...its only task in politics can be to explicate the feelings or emotions we are disponed to express in our normative political judgements. But that job may not have seemed very promising to many philosophers...» (PETTIT, 1993: 7).

La razón sustantiva del silencio, señala PETTIT, tiene que ver con lo incuestionable que aparecían para los filósofos analíticos — salvo para aquellos como POPPER que habían experimentado cuan atractivo puede ser el totalitarismo- valores tales como la libertad y la igualdad. La mayor parte de los filósofos habitaban
} 
Una característica de este modo de concebir la metaética ha sido su aspiración de neutralidad con relación a los problemas de moralidad sustantiva. El discurso metaético se presenta como un discurso de segundo orden, no comprometido con el discurso moral de primer orden. Pretende poseer dos tipos de neutralidad: referencial y predicativa. Una concepción metaética posee neutralidad referencial cuando lo que afirma acerca de la justificación o significado de los juicios morales se refiere y es compatible con todas las posiciones morales de primer orden (GEWIRTH, 1968: 215). La primera posición metaética con este tipo de neutralidad fue el Emotivismo, en el sentido de que lo que sostenía acerca de los juicios morales se aplicaba a todos ellos, sin discriminar acerca de su corrección o incorrección sustantiva ${ }^{6}$. Una concepción metaética posee neutralidad predicativa, en cambio, cuando el predicado que atribuye - y con el cual evalúa- a los juicios morales de primer orden no tiene carácter moral sino, lógico, epistemológico, ontológico, o de otro tipo (GEWIRTH, 1968: 2016) 7 .

Como hasta el modo más original de ser aburrido también termina aburriendo, la situación ha comenzado a cambiar también en las escuelas de derecho con una fuerte tradición positivista de España, Italia y Latinoamérica ${ }^{8}$. La discusión metaética ha perdido gradualmente espacio, salvo en ciertos reductos donde el escepticismo es predicado casi como un dogma. Gradualmente la reflexión ética sustantiva ha comenzado a ganar terreno y el ambiente académico no es tan hostil como solía serlo frente a la reflexión moral. Los juristas han vuelto a pensar que tienen algo para aprender y para decir acerca de los temas morales sustantivos.

un entorno «...where such values as liberty and equality and democracy held unchallenged sway. There were debates, of course, about the best means, socialist or otherwise, of advancing those values. But such debates would have seemed to most analytical philosophers to belong to the empirical social sciences. Hence those philosophers may not have seen any issues worth pursuing in the realm of political philosophy itself» (PETTIT, 1993: 8).

${ }^{6}$ Como señala Alan GEwIRT todas las filosofías morales han ofrecido una respuesta a la pregunta por el significado o la justificación de los juicios morales. Sin embargo, estas respuestas se aplicaban sólo a los juicios que consideraban correctos, no a todos los juicios morales con independencia de su contenido. En este sentido, sostiene, la neutralidad referencial es propia del discurso metaético que comienza con el Emotivismo. GEWIRT piensa que las reflexiones de los filósofos clásicos — como ARISTÓTELES- acerca del significado y la justificación de los juicios morales no poseía ni aspiraba a esta neutralidad. Adicionalmente, sostiene, esto también es cierto de filósofos como KanT y Hume, así como de la Filosofía Moral previa al Emotivismo. Señala: «...When the intuitionists discussed the intuitions by which they held that the meanings of basic moral concepts and the truth of basic moral propositions are grasped, they took as their "data" what they regarded as "the moral convictions of thoughtful and well-educated people" They did not think that the moral doctrines which, in their view, were untrue or incorrect, such as those of Hitler or Mussolini, were themselves grasped by intuition...» (GEWIRTH, 1968: 215)

7 Sólo este tipo de discurso metaético, que por otra parte es el que ha ganado adeptos en las escuelas de derecho con fuerte tradición positivista debido a su aspiración de neutralidad, es el que queda sujeto a la crítica de Williams que he formulado. La discusión sobre la justificación última de la moral llevada adelante por Thomas SCANLON, o la referida a la justificación de las teorías de la justicia sobre la base del equilibrio reflexivo desarrollada por RAWLS, para sólo poner dos ejemplos, no aspiran a ser sustantivamente neutrales y no poseen el problema que denuncio en el texto.

Aunque el uso del término metaética se ha ampliado para dar cabida también a reflexiones sustantivas como las antes citadas, sigue siendo dominante un modo de hacer metaética que aspira a la neutralidad. El Expresivismo de Allan GIBBARD y el Proyectivismo de Simon BLACKBURN, son ejemplos paradigmáticos de este enfoque. Como señala Ronald DwORKIN, los filósofos piensan que «...the most fundamental questions about morality are not themselves moral, but rather metaphysical, questions...» (DwORKIN, 2011: 25).

Agradezco a un evaluador anónimo el haberme hecho notar la necesidad de realizar las aclaraciones vertidas en esta nota.

${ }^{8}$ El cambio se había producido antes en las escuelas de derecho del mundo anglosajón. 
Que la discusión moral se haya tornado sustantiva, es algo beneficioso. Sin embargo, en todo cambio la transición implica riesgos y ha creado las circunstancias para que aparezca un segundo modo viciado de hacer Filosofía Moral. Un número creciente de académicos formados en los departamentos de filosofía de las escuelas de derecho, en donde sólo han recibido formación metaética, ha comenzado a hablar de problemas sustantivos. Movidos por intereses morales —en el mejor de los casos- o sólo para estar a la moda o dejar de aburrir — en el peor — se han abocado a la discusión moral y política. El resultado ha sido una mezcla de activismo político y lugares comunes.

Este segundo vicio aqueja mayormente a la Filosofía Moral que se hace en aquellas escuelas de derecho con una fuerte tradición positivista, donde el interés por la metaética ha evitado que sus académicos reciban cualquier formación en la reflexión ética sobre cuestiones sustantivas ${ }^{9}$. El problema radica en que aunque la formación técnica no es condición suficiente para hacer Filosofía Moral, «...(a)ny body who is going to be interested in moral philosophy must have a decent technical grounding, unless he or she happens to be a genius...» (WILLIAMS, 1983: 43).

La impaciencia de los juristas frente a estos dos modos viciados de hacer Filosofía Moral, que oscilan entre la preocupación por el significado de los términos morales y el diletantismo, está bien justificada. El aburrimiento que les provocan las interminables elucubraciones metaéticas o las prédicas repletas de obviedades carentes de todo rigor intelectual, no debe llamarlos a la reflexión. Es un síntoma auspicioso de salud mental.

El tercer vicio de la Filosofía Moral es uno sobre el que WiLLIAms trabajó extensamente. Básicamente WiLLIAMS señala que existe un problema con la aspiración de brindar una teoría omnicomprensiva de la moralidad que brinde una «...philosophical structure which, together with some degree of empirical fact, will yield a decision procedure for moral reasoning...» (WILLIAMS, 1981: x). El problema radica en que una teoría semejante indefectiblemente termina desconectándose de la experiencia moral. Peor aún, la teoría se erige como una especie de legisladora de los sentimientos morales.

Este modo de hacer Filosofía Moral — a diferencia del viciado por la metaéticase ocupa de los problemas concretos y - a diferencia de la torpe militancia- lo hace con instrumental filosófico. No obstante, aunque la técnica y la preocupación práctica son necesarias, no son suficientes. La Filosofía Moral debe comenzar en la experiencia moral y culminar en ella. Una reflexión filosófica que no parte de una conciencia agudizada del drama humano que subyace a los problemas morales y no está abierta a

\footnotetext{
9 Afortunadamente cada vez son más el número de escuelas de derecho que no padecen el sesgo escéptico y la obsesión metaética. Un indicio de ello es el número creciente de tesis doctorales que abordan asuntos sustantivos vinculados con la Filosofía Política, como, por ejemplo, la justificación de los derechos sociales, la evaluación moral de las instituciones jurídicas, derechos humanos o justicia global. La preocupación por los problemas formales vinculados con la sistematización del derecho o los problemas vinculados con la filosofía del lenguaje, aunque siguen estando presentes ha perdido el predominio que solía tener. Algunos de los departamentos de filosofía del derecho de estas escuelas cuentan hoy con personas sólidamente formadas en Filosofía Moral y Política. Un ejemplo paradigmático es el departamento de filosofía del derecho de la Universidad Pompeu Fabra, que en la actualidad cuenta con un potente grupo de Filosofía Política. Lo mismo es cierto de otros centros de altos estudio como la Universidad de Alicante o la Universidad Autónoma de Madrid, por mencionar sólo los casos más notables en España.
} 
variar sus conclusiones por más alejadas que se encuentren de la experiencia moral, es un modo viciado de reflexión.

El vicio ha dado lugar a un modo particular de hacer lo que ha venido a denominarse «filosofía aplicada». Consiste en tomar un conjunto de principios morales generales y pasarlos como un tamiz por todos los problemas morales para obtener respuestas. Primero se resuelven los asuntos de importancia teórica, y luego con los principios generales a mano se desciende a aplicarlos a los problemas concretos. Como ya las cuestiones de importancia han sido resueltas no existe nada en el momento de la aplicación que pueda hacer variar los principios generales. Se trata de un modo cuasi-legalista de hacer Filosofía Moral. Esto ha permitido, según Williams que «...(a)ll the philosophical journals are full of issues about women's rights, abortion, social justice, and so on. But an awful lot of it consists of what can be called in the purely technical sense a kind of casuistry, an application of certain moral systems or principles or theories to discussing what we should think about abortion...» (WILLIAMS, 1983: 44).

WiLLIAMS pone un ejemplo de este modo de reflexión viciado que lamentablemente se asemeja demasiado al tipo de reflexión moral que puede encontrarse en algunos de los seminarios sobre Filosofía Moral que se dictan en las escuelas de derecho. Señala:

...I recall a notorious article written by a moral philosopher who argues that if abortion is legitimate, then so is infanticide. That is an inference which Catholics have used in the past, but they have always used it to show that abortion was wrong. He uses it to show that infanticide is permissible. I regard his argument as simply fallacious, unsound. But the point is that there is something mildly grotesque about the idea that one can arrive at a conclusion of that human magnitude by pushing around a few quick arguments in a philosophical journal. There is just something odd about that. The moral philosopher has to be reflective about the kind of discourse he is engaged in, the kind of dialogue he is engaged in with the public (WILLIAMS 1983: 44) ${ }^{10}$.

Como señala WiLLIAMS, existe algo grotesco en que alguien pueda concluir a partir de unas cuantas premisas que el infanticidio está moralmente justificado. Lo grotesco es que pueda llegarse a una conclusión de tal «magnitud humana» sin que esto provoque la revisión de algunas premisas del argumento. Lo inaudito es que la conclusión no funcione como una reducción al absurdo del argumento completo. Es extravagante que pueda concluirse a partir de unas pocas premisas que el infanticidio es permisible - tal como decía el artículo que WiLLiams tuvo ocasión de leer- o que el aborto o la extinción de los animales carnívoros es un deber moral ${ }^{11}$ — tal como he tenido ocasión de escuchar-.

${ }^{10}$ Se trata del artículo de Michael ToOLEy, «Abortion and infanticide» (1972), que Williams luego menciona y critica en Ethics and theLimits of Philosophy (WILLIAMS, 1985: 112).

${ }_{11}$ Tanto la conclusión de que el infanticidio es permisible como la de que existe un deber moral de provocar la extinción de ciertas especies carnívoras, fueron pensadas originalmente como reducciones al absurdo de las premisas que las sostenían. En este último caso, el primero en extraer esta conclusión como una reducción al absurdo de la premisa que los animales poseían derechos, fue David RITCHIE a finales del siglo XIX. Éste señalaba: «...Well, then, in our exercise of our power and in our guardianship of the rights of animals, must we not project the weak among them against the strong? Must we not put to death blackbirds and thrushes because they feed on worms, or (if capital punishment offends our bumanitarianism) starve them slowly by permanent captivity and vegetarian diet? What becomes of the "return to nature" if we must prevent the cat ss nocturnal wanderings, 
Aunque la crítica de WiLliams se dirige a todo tipo de teoría moral, creo que una vez que uno advierte las razones que subyacen a la misma es posible afinarla precisando su objetivo. El blanco de la crítica son aquellas formas de reflexión moral que no reconocen que ciertas prácticas de ofrecer consideraciones como razones morales no necesitan ser respaldadas a su vez por razones morales más generales. Si uno se embarca en esta búsqueda lineal de razones — sostiene WILLIAMS — «...there will have to be at least one practice of reason-giving for which no reason is given and which holds itself up. Looked at in one way, this result may encourage the simplification principle I mentioned before: if having an unrationalized principle is irrational, it is good to have as little irrationality as possible» (WILLIAMS, 1985: 113). Quien realiza esta inferencia, no obstante, está en un error. No ha advertido el carácter central de algunas de nuestras experiencias morales.

Esto no implica que el criterio para decidir qué convicciones morales son correctas deba apelar a la «opinión pública», o a lo que la mayoría considera correcto. La mayoría puede ser tan insensible a la «magnitud humana» de los problemas morales o tan irreflexiva en sus convicciones, como quien realiza el tipo de Filosofía Moral que acertadamente WilLIAMS critica. El error denunciado por WiLLIAMS no consiste en que los filósofos morales que critica no realicen encuestas de opinión ${ }^{12}$. Tampoco consiste en una mera acusación de regreso al infinito. El error reside en creer que una convicción moral es irreflexiva, y no puede ser utilizada como una razón, a menos que exista una razón para utilizarla de esta manera. Lo equivocado es desgajar la reflexión moral de la experiencia moral, para concluir que toda creencia moral que no puede justificarse a partir de una razón ulterior es por ese mero hecho un prejuicio que no debe ser atendido ${ }^{13}$.

Este modo erróneo de concebir cuando una creencia moral está injustificada conduce de modo natural a una concepción fundacionalista de la justificación moral. Toda creencia moral — para no ser irreflexiva y prejuiciosa — debe estar justificada en otra creencia o principio moral de mayor grado de generalidad. Mientras más general es un principio, más fundamental o importante es el valor al que se refiere. Cuando un principio entra en conflicto con una creencia moral particular — por ejemplo, que matar a éste niño es inmoral o provocar la extinción indolora de este animal carnívoro es inco-

lest she should wickedly slay a mouse? Are we not to vindicate the rights of the persecuted prey of the stronger?...» (RiTCHIE, 1894: 109).

12 Adam SWIFT ha criticado de modo acertado esta apelación a la «opinión pública» para validar los juicios morales acerca de la justicia. SWIFT sostiene que ésta es —en parte- la posición defendida por David MiLleR. SWIFT piensa que esta «relación constitutiva» entre la «opinión pública» y la validez de los juicios de justicia es falsa. No obstante piensa que existen otros tipos de vinculación que son válidos. La «opinión pública» es relevante para determinar si es posible llevar una concepción de justicia a la práctica, para determinar si sería legítimo hacerlo, o para determinar el contenido de las exigencias de justicia si los principios de justicia correctos son deferentes con las expectativas de los individuos (SWIFT, 2002: 13-28).

${ }_{13}$ Señala WiLliams al respecto: "Prejudice" is a powerful and ambiguous word, and its relations to theory are equally ambiguous. It has played a large role in the Cartesian tradition, in which any belief counts as a prejudice that has not yet been given a foundation. In this sense, it is certainly contrasted with theory, but in this sense, as I have already said, everything is a prejudice, in science as in ethics. In another and narrower sense, it means any belief one holds only because one has not reflected on it. In this sense, it may well be that we inevitable bave prejudices, but at any rate the reflection demanded, which some beliefs will survive, need not be the reflections of ethical theory...» (WILLIAMS, 1985: 117). 
rrecto- es la intuición moral particular la que debe ceder, a menos que encontremos un principio general que la justifique. Sólo en este caso la profundidad de los valores en conflictos será comparable.

Esta deriva fundacionalista posee dos variantes. La primera, más extrema, niega a las creencias o intuiciones morales particulares cualquier valor. Éstas no tienen siquiera valor epistémico para descubrir cuáles son los principios morales correctos. Los principios morales son auto-evidentes — de modo que no es necesario apelar a las convicciones morales particulares para de allí elevarse a los principios generales que las justifican-y las creencias morales particulares sólo están justificadas si derivan de estos principios generales. Peter SINGER, un cultor de este modo de hacer Filosofía Moral, deja clara su concepción cuando afirma que un filósofo moral debería «... search for undeniable fundamental axioms; build up a moral theory from them; and use particular moral judgments as supporting evidence, or as a basis for ad hominem arguments, but never so as to suggest that the validity of the theory is determined by the extent to which it matches them...» (SINGER, 1974:517) ${ }^{14}$.

La segunda concede a las creencias morales particulares un rol epistémico. Las intuiciones morales son irreflexivas e injustificadas a menos que estén respaldadas por un principio general que las funde. En esto no se diferencia de la posición anterior. Tampoco se diferencia en la mayor importancia que concede a los principios generales frente a las creencias morales particulares, cuando entran en conflicto. Jeff McMAHAN, un defensor de esta segunda variante, afirma que «...the principles seem to be epistemically more basic, more secure. They articulate our core values which unify, explain, and justify our intuitive judgments... In short, the principles are foundational with respect to the intuitions...» (MCMAHAN, 2013: 114). Sin embargo, se diferencia de la posición anterior en tanto sostiene que «...it does not regard the foundational principles as self-evident...» (MCMAHAN, 114). Los principios fundacionales son descubiertos a través de un proceso de equilibrio reflexivo cuyo punto de partida son las intuiciones o creencias morales particulares (MCMAHAN, 2013: 111) ${ }^{15}$.

Lo relevante es que ambas posiciones comparten la idea errónea de que es irreflexivo e injustificado tomar algo como una razón, a menos que exista una razón para utilizarla de esta manera. El tipo privilegiado de reflexión moral es de orden subsustivo. De acuerdo con esta visión, «(w)hen one's moral intuition is challenged by another person, it is natural to respond by appealing to claims of a higher level of generality that imply or explain the intuition. The assumption is that the credibility of the intuition is enbanced if it can be subsumed under a plausible moral principle...». De acuerdo con la visión, el principio general nos da razones para tomar como una razón el contenido de nuestra intuición particular ${ }^{16}$.

14 Peter SINGER sostiene que este método fundacionalista es el utilizado por Henry SIDGWICK. Disputa así la afirmación de RAWLS en $A$ Theory of Justiceen el sentido que SIDGWICK utilizaba como método de justificación el equilibrio reflexivo.

15 Aunque este modo de hacer Filosofía Moral se aleja de lo que WiLliams denominaba «filosofía aplicada», comparte con ésta la convicción de que sólo es racional o reflexivo considerar a algo como una razón si existen razones más generales para hacerlo.

16 Agradezco a un evaluador anónimo el haberme hecho notar la necesidad de introducir estas precisiones. 
Un ejemplo puede ayudar. Supongamos que tengo la intuición de que es moralmente incorrecto matar seres humanos. Supongamos, adicionalmente, que alguien cuestiona que el hecho de que algo sea un ser humano es una razón para no matarlo. Según este modo viciado de hacer Filosofía Moral tal desafío me obligaría a buscar un principio más general que justificase la intuición particular que tengo en relación con los seres humanos ${ }^{17}$. Por ejemplo, uno que señalase que es moralmente incorrecto matar seres sintientes. Si no puedo encontrar un principio general que respalde mi intuición con respecto a los seres humanos, entonces afirmar que la mera humanidad es una razón para no matar es una afirmación irreflexiva. No es más que la manifestación de un tipo de prejuicio especista ${ }^{18}$.

Una vez que se abandona la idea errónea de que es irreflexivo tomar algo como una razón a menos que existan razones para hacerlo - y la necesidad de unificar la moral en principios generales (en el límite, un único principio)_- desaparece la falsa conexión entre generalidad e importancia o profundidad. Las creencias morales particulares no son irreflexivas por el mero hecho de que no pueda brindarse un principio general en su respaldo, y los principios generales no poseen indefectiblemente mayor importancia que las creencias morales particulares ${ }^{19}$. El carácter reflexivo de una creencia no le viene dado por el hecho de estar justificada en otra creencia general que corporiza un valor moral de mayor importancia. A riesgo de decir lo obvio, es necesario señalar que una creencia es reflexiva cuando ha sido objeto de reflexión, esto es, cuando ha sido examinada y no se han encontrado razones para sospechar que ha sido generada por factores que hagan dudar de su corrección, tales como el auto-interés, la indoctrinación, la manipulación, la presión de grupo, etcétera.

${ }_{17}$ Es necesario enfatizar que lo señalado en el texto no implica afirmar que todo tipo de fundacionalismo es un modo viciado de hacer Filosofía Moral. Lo viciado es la idea de reflexión subsuntiva que conduce a apartarse de la experiencia moral en busca de principios cada vez más generales. Aunque este modo de concebir a la reflexión moral conduce naturalmente al fundacionalismo, esto no significa que sea la única manera de llegar a él. Pueden brindarse razones a favor del fundacionalismo que no tengan nada que ver con este modo viciado de concebir la reflexión moral.

${ }^{18}$ Utilizo este ejemplo debido en parte a que es uno al que el mismo WiLLIAms hace referencia. Williams pensaba que era absurdo sostener que existía algo como el prejuicio especista, creo que en parte por las razones que ofrezco en el texto. Su posición sobre este asunto puede cotejarse en Williams. Para ver detalladamente la posición de WiLLiams sobre este asunto vid. WiLliams, 2006.

19 Este modo de concebir las intuiciones morales, como dotadas de una importancia comparable a la de los principios generales, es acorde con el modo en que RAWLS entiende la justificación moral. Tal como RAWLS entiende el equilibrio reflexivo, a diferencia de como lo hace MCMAHAN, éste debe incluir «...our considered convictions at all levels of generality; no one level, say that of abstract principles or that of particular judgments in particular cases, is viewd as foundational. They all may have an initial credibility...» (RAWLS, 1993: 8). Más adelante agrega «...it is a mistake to think of abstract conceptions and general principles as always overriding our more particular judgments...» (RAWLS, 1993: 45).

Este es un punto sobre el que el pensamiento de RAWLS ha ido evolucionando. El papel concedido a los juicios o intuiciones morales particulares es mucho más importante en sus primeros escritos. Allí RAWLS proponía un procedimiento para validar principios generales cuyo punto de partida era seleccionar los juicios morales particulares que pudiesen pasar el test de la reflexión. Luego se pasaba a obtener principios generales cuya aplicación a los casos concretos diese como resultado los juicios particulares de los cuales se había partido (RAWLS, 1951).

Por lo que respecta al método del equilibrio reflexivo, cabe destacar que WiLLIAms también suscribe la idea de que se trata de la herramienta adecuada en materia moral. Así, afirma que «...to the extent that we are thinking about the systematic representation of the content of ethics, the right method is something like reflective equilibrium... I think that that aspect of Rawls's project is admirable...» (WILLIAMS, 1999: 245). 
No hay nada que garantice que los principios generales sean más inmunes a este tipo de factores distorsionantes que lo que son las creencias morales particulares. Así, un principio tan general como el que sostiene que el carácter sintiente de un ser posee relevancia moral con independencia de si éste es o no un ser humano, puede perfectamente haber sido adoptado fruto de un mecanismo espurio. Si existe un grupo unido por este credo, alguien podría haber llegado a suscribirlo como un modo de lograr aceptación y contención. O podría tratarse de una manera de cobrar notoriedad en un ambiente donde las posiciones más extremas son las más escuchadas. O de una manifestación de desprecio y resentimiento hacia otros seres humanos. O de un afán de verse como superior moralmente a la mayoría que no acepta esta posición extrema ${ }^{20}$.

Elevarse de las creencias morales particulares a principios generales no es equivalente a reflexionar. Menos aún lo es ser insensible a la experiencia moral. La impaciencia de los juristas con este modo de hacer Filosofía Moral no debe ser corregida ni revisada. Lo que debe ser corregido es esta manera de hacer Filosofía Moral que, en palabras de WILLIAMS, «... it becomes so ar idly simplified that it really does not help people to think very well» (WILLIAMS, 1983: 44). Tal vez la impaciencia de los juristas no se debe a su carácter irreflexivo, o a su falta de conocimiento del instrumental filosófico, como usualmente piensa el filósofo moral incomprendido para reforzarse en su posición. Tal vez su impaciencia se debe a que no son indiferentes a la «magnitud humana» de las conclusiones que se extraen, o de lo que públicamente se afirma ${ }^{21}$.

\section{LAS VIRTUDES DE LA REFLEXIÓN JURÍDICA}

De los tres vicios que he señalado - la obsesión metaética, el activismo diletante y el distanciamiento de la experiencia moral - sin duda el último es el más peligroso. La tendencia humana a ocuparnos de los problemas sustantivos e importantes, y la preocupación de los juristas por los problemas prácticos, se presenta como un antídoto lo suficientemente potente contra la obsesión metaética. Prueba de tal hecho es que la reflexión sustantiva ha ido ganando cada vez más espacio y es de esperar que esta tendencia que comenzó en el ámbito de la Filosofía continúe avanzando en los departamentos de Filosofía de las escuelas de derecho. En aquellos lugares donde hoy se mantiene la obsesión metaética se debe más a circunstancias históricas y personales que a razones teóricas ${ }^{22}$.

20 Refiriéndose a quienes defienden la posición anti-especista, y en consonancia con el tipo de explicaciones que sugiero en el texto, WiLliams señala: «In many, more limited, connections hopes for self-improvement can lie dangerously closet to the risk of self-hat red. When the hope is to improve bumanity to the point at which every aspect of its bold on the world can be justified before a bigher court, the result is likely to be either selfdeception, if you think you have succeeded, or self-batred and self-contempt when you recognize that you will always fail. The self-hatred, in this case, is a batred of humanity...» (WILLIAMS, 2006: 152).

21 Este tercer vicio debe ser distinguido de la insensibilidad de los principios morales a las consideraciones empíricas. Existe actualmente un profuso debate alrededor de la relevancia de lo que denominan teorías ideales. El defecto que se pone de manifiesto en el texto no está vinculado con la insensibilidad a datos empíricos sino con la insensibilidad a ciertas intuiciones morales.

22 José Juan MORESo ha sido uno de los juristas que con más ahínco ha combatido esta obsesión en el seno de las escuelas de derecho. Específicamente ha dedicado sus esfuerzos a mostrar que no existe ninguna conexión conceptual entre positivismo jurídico, relativismo moral y liberalismo político o tolerancia (MORESO, 2012; 2013). 
El diletantismo activista es más arriesgado que el anterior vicio porque ocupa el espacio que la reflexión moral y política debería tener. Mientras en el caso anterior no se lleva adelante reflexión sustantiva, en este caso se abordan los problemas sustantivos pero sin ninguna reflexión. El riesgo reside en que los juristas terminen convencidos de que la Filosofía Moral es ese conjunto de trivialidades y admoniciones que el diletante tiene para decir. No obstante, los juristas más que nadie están adiestrados para identificar sofistas y farsantes. Parte de su impaciencia frente a este modo de hacer Filosofía Moral creo que se debe a esto.

El tercer vicio, la falta de sensibilidad a la experiencia moral, es más difícil de erradicar. Sin embargo, es aquí donde la reflexión jurídica tiene más que aportar a modo de antídoto. En primer lugar, ha sido el derecho y la reflexión jurídica la que ha tenido que dar respuesta a los problemas prácticos de las sociedades humanas. Durante casi cuatro mil ${ }^{23}$ años los juristas han tenido que enfrentarse a los problemas sustantivos - aborto, eutanasia, responsabilidad por daño, etc.- en primera persona. Esto los posiciona mejor que nadie para percibir la «magnitud humana» de los problemas que enfrentan.

Los juristas en sus diferentes áreas — derecho civil, de familia, ambiental, etc.— se enfrentan a diario al mismo drama humano que confronta la Filosofía Moral. Los problemas prácticos de personas de carne y hueso han desfilado durante siglos frente a sus ojos. Es esta inmediatez con los problemas prácticos lo que les permite advertir lo que algunos filósofos morales no pueden. Es esta situación la que ha agudizado su sensibilidad y los vuelve impacientes con soluciones completamente alejadas de la experiencia humana. Quien a diario trabaja con problemas familiares no puede concebir que se argumente a favor del deber general de abortar. Quien a diario se enfrenta a problemas ambientales reales se impacienta cuando se le dice que existe el deber general de extinguir especies. Su sensibilidad frente a la complejidad y magnitud del problema le impide tomarse en serio estas soluciones planas.

En segundo lugar, los juristas han sido investidos con una responsabilidad práctica. No sólo deben enfrentar problemas reales sino que además deben ofrecer soluciones. El objetivo principal de la reflexión de los juristas no es publicar en revistas especializadas o sorprender a su mentor. Su objetivo es encontrar la solución adecuada para un caso que tiene la responsabilidad de resolver. Esta reflexión responsable que soluciona los problemas teóricos con la mirada puesta en la realidad, puede servir para corregir la despreocupación de cierta Filosofía Moral por el carácter contraintuitivo o fantástico de sus conclusiones. La necesidad de ofrecer soluciones concretas a problemas reales - que caracteriza a los juristas y que padeció THOMSON— tal vez pueda, después de todo, funcionar como una virtud.

Por último, existe algo en el estilo de la reflexión jurídica que puede servir para corregir el distanciamiento entre la reflexión y la experiencia moral. En su intento de remontarse a los primeros principios de la moralidad, el razonamiento moral corre el riesgo de perder el hilo conductor que lo conecta con la experiencia moral que le sirve de punto de partida. La reflexión sobre la experiencia moral humana al ascender en

${ }^{23}$ Tomo como dato de referencia la fecha en la que se ubica temporalmente al Código de Hamurabi (aproximadamente $1770 \mathrm{AC}$ ). 
su nivel de abstracción corre el riesgo de desfigurarla hasta perder de vista su carácter humano. La reflexión jurídica posee una deferencia hacia la experiencia pasada que puede servir de contrapeso a este vuelo ascendente.

La reflexión jurídica mira hacia la experiencia humana acumulada en el pasado. Presupone que hay algo valioso - aunque sea en germen - en las respuestas que otros seres humanos han dado de modo reflexivo a los problemas humanos. Los juristas son entrenados en esta forma de argumentar. Por supuesto, esta forma de argumentación corre el riesgo de consagrar el statu quo y por ello necesita del acicate de la reflexión filosófica. Sin embargo, también representa una virtud: la reflexión jurídica es, por decirlo de algún modo, humilde; es una empresa humana colectiva, enraizada en la experiencia.

Para la Filosofía Moral viciada a la que he hecho referencia, por el contrario, el solipsismo es una virtud. Mientras más solo se encuentre este tipo de filósofo moral en sus posiciones, mayor es su convicción de que ha llegado a los más altos principios morales donde nadie antes que él ha tenido el coraje o la inteligencia de ascender. Su soledad —esto es, el carácter contraintuitivo de sus conclusiones- se transforma en la prueba de su éxito. En las cumbres siempre se está solo. A lo sumo lo que queda es reunirse con otros pocos que han logrado alcanzar las mismas alturas. Este tipo de reflexión moral, por utilizar un nombre, es ambiciosa.

Pienso que el carácter humilde de la reflexión jurídica puede servir de paliativo a la ambición de este tipo de Filosofía Moral. Puede contribuir a que el filósofo moral deje de ver el carácter extremo de sus conclusiones como una virtud. Puede ayudar a que deje de percibir su aislamiento como prueba de su éxito. Puede favorecer que la Filosofía Moral vuelva a tener contacto con la experiencia moral que la nutre. Tal vez viendo el estilo deferente a la experiencia que posee la reflexión jurídica, este tipo de filósofo moral descubra que existe un estilo deferente a la experiencia moral de hacer Filosofía.

Una vez más, no se trata de crear un tipo de jurisprudencia filosófica que apela a la autoridad de las opiniones del pasado o a las opiniones comunes del presente. Simplemente se trata de recordar a este tipo de filósofos morales lo que Henry SIDGWICK —alguien por otra parte interesado también en elevarse al principio último de la moralsostenía. Según él, identificar lo moralmente correcto no puede quedar exclusivamente en manos de los filósofos morales «...because their moral judgment on any particular question of duty, even supposing them to have obtained all available information as to the particular facts of the case, is not to be trusted, unless it is aided, checked, and controlled by the moral judgment of persons with less philosophy but more special experience» (SIDGWICK, 1893: 7). Pues bien, nadie con más experiencia en abordar los problemas prácticos que los juristas.

El servicio que los juristas pueden prestarle a la Filosofía Moral era destacado por DwORKIN en la conferencia a la que he hecho referencia al inicio de este trabajo. La importancia de este servicio se vuelve evidente una vez que se advierte lo pernicioso del vicio que puede corregir. DwORKIN finalizaba su conferencia señalando que los juristas están capacitados para percibir con mayor agudeza las implicaciones de los principios morales y políticos, y para considerar las cuestiones morales en contextos 
reales (DwORKin, 2000: 14). Aunque su consejo de prestar atención a la Filosofía Moral ha sido seguido por los juristas, no estoy seguro que haya sucedido lo mismo con el consejo en sentido inverso dirigido a los filósofos morales.

\section{CONCLUSIÓN}

Existen diferentes servicios que los filósofos morales pueden prestarles a los juristas. En primer lugar, la Filosofía Moral —al menos la analítica - puede enseñar a los juristas las herramientas de análisis y mostrarle su uso para abordar problemas prácticos. La Filosofía Moral y la reflexión jurídica comparten parcialmente el instrumental técnico y el contacto entre una y otra puede ser beneficioso. En segundo lugar, el carácter pausado de la reflexión moral — no acuciada por urgencias institucionalespuede ayudar a paliar la tendencia de los juristas a preocuparse sólo por la búsqueda de soluciones. Finalmente, en tercer lugar, la reflexión jurídica puede beneficiarse de las conclusiones sustantivas a las que han arribado los filósofos morales sobre temas prácticos. Los problemas que han ocupado a la Filosofía Moral y al derecho, después de todo, son semejantes.

Hay, no obstante, tres maneras viciadas de hacer Filosofía Moral frente a las cuales los juristas han hecho bien en mostrarse impacientes. La primera consiste en la obsesión metaética fundada en un sesgo escéptico hacia cualquier reflexión sustantiva. La segunda es el diletantismo activista de aquellos que sin ninguna preparación teórica se dedican a hablar de problemas morales y políticos. La tercera consiste en una forma de reflexión filosófica que en su afán de encontrar los principios supremos ha perdido todo contacto con la experiencia moral humana.

El contacto con los juristas puede ayudar a combatir estos vicios, prestando de este modo la reflexión jurídica un servicio a la Filosofía Moral. Aunque la obsesión metaética, como he señalado, ha caído por su propio peso, el contacto con los juristas puede contribuir activamente a su definitivo descenso. Los juristas están ávidos de encontrar soluciones a problemas sustantivos, lo que es incompatible con la «dieta lingüística» que los obsesionados con la metaética le ofrecen.

Aunque tampoco representa un gran riesgo, el diletantismo activista también puede ser combatido por el contacto con los juristas. Este tipo de diletante tiende a presentarse frente a los filósofos como un jurista y frente a los juristas como un filósofo. Los filósofos le perdonan su falta de preparación técnica porque imaginan que lo que tiene para decir posee alguna relevancia jurídica. Los juristas suelen escucharlo porque se presenta como un filósofo. Nada mejor que unos y otros converjan para que se vuelva evidente que el diletante no es ni filósofo ni jurista.

Finalmente, y lo que es más importante, el contacto con los juristas puede ayudar a corregir el vicio más pernicioso de la Filosofía Moral. Puede ayudar a que los filósofos morales tengan una conciencia agudizada de la «magnitud humana» de los problemas que enfrentan y las soluciones que proponen. La reflexión jurídica, dado su carácter situado, urgido y responsable, puede contribuir a que los filósofos morales no pierdan contacto con la primera experiencia de ser humanos en su afán por encontrar los principios últimos. 
El contacto con los juristas puede ayudar a que los filósofos morales no sucumban a la tentación de creer que el hecho de que no puedan encontrar razones para que algo cuente como una razón determina que deban dejar de considerarlo una razón so pena de irracionalidad. Este error ha llevado a los filósofos morales a despojarse de las experiencias morales más básicas. Así, por ejemplo, que no podamos esgrimir ninguna razón para considerar que matar a un ser humano es inmoral por el mero hecho de que se trata de un ser humano no hace que debamos abandonar la idea de que la humanidad es valiosa. Tal vez, como señala WILLIAMS, esto simplemente prueba que el valor moral de la humanidad «... is more directly convincing than any reason that might be advanced for it... is more convincing as a reason than any reason which might be advanced for its being a reason...» (WILLIAMS, 1981: 81).

\section{BIBLIOGRAFÍA}

ABA, Section of Legal Education and Admission to the Bar, 1992: Legal Education and Professional Development - An Educational Continuum-. Report of the Task Force on Law Schools and the Profession: Narrowing the Gap.

Dworkin, R., 2000: «Must Our Judges Be Philosophers? Can They Be Philosophers?», conferencia ofrecida en ocasión de recibir la distinción de Scholar of the Year por el New York Council for the Humanities. El texto original de la conferencia puede cotejarse en bttp:// www.culturefront.org/culturefront/Dworkin.html. Versión castellana en Estudios de Derecho, vol. LXIV., núm. 144, diciembre de 2007.

- 2011: Justice for Hedgehogs, Cambridge, Mass.: Harvard University Press.

GEWIRTH, A., 1968: «Metaethics and Moral Neutrality», Ethics, 78: 214-225.

McMahan, J., 2013: The Blackwell Guide to Ethical Theory, La Follete, Hugh y Persson, INGMAR (eds.), Oxford: Wiley-Blackwell.

Moreso, J. J., 2012: «Positivismo Jurídico, Relativismo Moral y Liberalismo Político», Teoría Politica, 2.

- 2013: «Donde la Pala se nos Dobla: de Nuevo Sobre Metaética y Política», Teoría Política, 3.

Nussbaum, M., 1993: «The Use and Abuse of Philosophy in Legal Education», Stanford Law Review, 45: 1627-1645.

PetтtT, Ph., 1997: «Analytical Philosophy», en A Companion to Contemporary Political Philosophy, R. Goodin, P. PetTit y T. Pogge (eds.), Cambridge, Mass.: Blackwell, 5-35.

Posner, R., 1993: Overcoming Law, Cambridge, Mass.: Harvard University Press.

Rawls, J., 1951: «Outline of a Decision Procedure for Ethics», Philosophical Review, 60: 177-197.

- 1993: Political Liberalism, Expanded Edition, New York: Columbia University Press.

Ritchie, D. G., 1894: Natural Rights, London: Swan Sonnenshein.

SIDGWICK, H., 1893: «My Station and Its Duties», International Journal of Ethics, 4: 1-17

SingeR, P., 1974: «Sidgwick and Reflective Equilibrium», The Monist, 58: 490-517.

SwIFT, A., 2002: «Social Justice: Why Does it Matter What the People Think», en D. BEEL y A. DE-Shalit (eds.), Forms of Justice: Critical Perspectives on David Miller's Political Philosophy, Lanham: Rowman \& Littlefield Publishers.

Tooley, M., 1972: «Abortion and Infanticide», Pbilosophy and Public Affairs, 2: 37-65. WILLiAmS cita la versión presente en M. COHEN, Th. NAGEL y Th. SCANLON (1983), The Rights and Wrongs of Abortion, Princeton: Princeton University Press. 
Williams, B., 1972: Morality, Cambridge: Cambridge University Press.

- 1981: Moral Luck, Cambridge: Cambridge University Press.

- 1983: «The uses of Philosophy» (entrevista realizada por Donald MCDONALD), The Center Magazine, noviembre/diciembre 1983: 40-49.

- 1985: Ethics and the Limits of Philosophy, London y New York: Routledge.

— 1999: «Seminar with Bernard Williams», Ethical Perspectives, 6: 243: 265.

- 2006: Philosophy as a Humanistic Discipline, Princeton y Oxford: Princeton University Press. 